\title{
Maternal Periconceptional Folic Acid Supplementation and the Risk of Non-Syndromic Oral Clefts in Offspring: A Population-Based Case-Control Study in Chengdu, China
}

\author{
Wenli Xu \\ West China Second University Hospital, Sichuan University, Chengdu, Sichuan \\ Ling Yi \\ West China Second University Hospital, Sichuan University, Chengdu, Sichuan \\ Changfei Deng \\ West China Second University Hospital, Sichuan University, Chengdu, Sichuan
}

\section{Ziling Zhao}

Sichuan Provincial Hospital for Women and Children, Affiliated Women and Children's Hospital of Chengdu Medical College

\section{Tianjin Zhou}

Sichuan Provincial Hospital for Women and Children, Affiliated Women and Children's Hospital of Chengdu Medical College

\section{Gang Zhang}

Sichuan Provincial Hospital for Women and Children, Affiliated Women and Children's Hospital of Chengdu Medical College

\section{Longrong Ran}

Chengdu Women's \& Children's Central Hospital, Chengdu, Sichuan

\section{Zhihong Ren}

Chengdu Women's \& Children's Central Hospital, Chengdu, Sichuan

\section{Shunxia Zhao}

Chengdu Women's \& Children's Central Hospital, Chengdu, Sichuan

\section{Hanmin Liu}

Pediatric Department, West China Second University Hospital, Sichuan University, Chengdu, Sichuan

\section{Li Dai ( $\nabla$ daili@scu.edu.cn )}

Key Laboratory of Birth Defects and Related Diseases of Women and Children (Sichuan University), Ministry of Education, Chengdu, Sichuan

\section{Research Article}

Keywords: Oral clefts, folic acid, population-based case-control study

Posted Date: January 8th, 2021

DOI: https://doi.org/10.21203/rs.3.rs-136950/v1

License: (c) (i) This work is licensed under a Creative Commons Attribution 4.0 International License. Read Full License 


\section{Abstract}

Maternal periconceptional folic acid supplementation (FAS) has been documented to be associated with decreased risk of nonsyndromic oral clefts (NsOC). However, the results remain inconclusive. In this population-based case-control study of 807 singletons affected by NsOC and 8070 healthy neonates who were born between October 2010 and September 2015 in Chengdu, China, we examined the association of maternal FAS with the risk of nonsyndromic cleft lip with or without cleft palate (NsCL/P), and cleft palate (NsCP). Unconditional logistic regression analysis was used to estimate the crude and adjusted odds ratios (ORs) and $95 \%$ confidential intervals $(\mathrm{Cl})$. Significant associations were found between maternal periconceptional FAS and decreased risk of NsCL/P ( $\mathrm{aOR}=0.41,95 \% \mathrm{Cl}: 0.33-0.51)$. This protective effect was also detected for NsCL (aOR = 0.42, 95\% Cl: $0.30-0.58)$ and NsCLP (aOR=0.41, 95\% Cl: 0.31-0.54). Both maternal FAS started before and after the last menstrual period (LMP) were negatively associated with NsCL/P (before LMP, aOR $=0.43,95 \% \mathrm{Cl}$ : $0.33-0.56$; after LMP, aOR $=0.41,95 \% \mathrm{Cl}$ : 0.33-0.51). The association between NsCP and maternal FAS initiating before LMP was significant $(\mathrm{aOR}=0.52,95 \% \mathrm{Cl}$ : $0.30-0.90)$, but the statistical power seemed weak due to limited number of NsCP cases. The findings suggest that maternal periconceptional FAS can reduce the risk of each subtype of NsCL/P in offspring, while the potential effect on NsCP needs further investigations.

\section{Introduction}

Oral clefts $(\mathrm{OC})$, resulting from the incomplete fusion of primary palate and/or secondary palate during the 7th to 10th week of pregnancy, usually include syndromic or nonsyndromic cleft lip with or without cleft palate (CL/P) and cleft palate (CP). ${ }^{1}$ Depending on geographic regions and races, oral clefts affect 1 in 500 to 2500 births. ${ }^{1}$ The nonsyndromic clefts (NsOC) are one of most common birth defects in China. ${ }^{2}$ The reported prevalence of NsOC can be as high as 1.8/1000 in some provinces of China, causing huge disease burden on family and society. ${ }^{2-4}$

Maternal folic acid supplementation (FAS) before and during early pregnancy is a well-established prevention approach for neural tube defects (NTDs). As facial mesenchyme is derived from neural crest, the protective effect of maternal FAS on oral clefts has been studied in various populations, but the findings are inconclusive. ${ }^{5-8}$ Most observational studies conducted in Europe, ${ }^{9-14}$ America, ${ }^{15-17}$ and Asia ${ }^{18-21}$, have shown that maternal FAS during early pregnancy is associated with decreased risk of nonsyndromic $\mathrm{CL} / \mathrm{P}(\mathrm{NsCL} / \mathrm{P})$, while other investigations in these areas did not. ${ }^{22-27}$ Some of them revealed a preventive effect for nonsyndromic cleft palate (NsCP), ${ }^{9,13-15}$, whereas a few studies suggested maternal FAS or multivitamin intake as risk factor of NsCL/P and NsCP. ${ }^{28}$ Though two recent meta-analyses support the potential preventive effect of maternal FAS against $\mathrm{NsCL} / \mathrm{P}$ and $\mathrm{NsCP},{ }^{7,8}$ the currently available evidence is insufficient and conflicting, particularly for some cleft subtypes. ${ }^{29-31}$ In fact, population-based evidence from China is relatively scarce. With the exception of one cohort in three provinces, ${ }^{18}$ a population-based case-control study in Shenyang city, ${ }^{21}$ and a propensity-matched study in Shaanxi province, ${ }^{26}$ almost all the observational studies in China have been hospital-based. $32-36$

Chengdu, the capital of Sichuan province, is a megacity located in the southwest of China where the prevalence of OCs is higher than the national average. ${ }^{3}$ Following the Guide of National Folic Acid Supplementation Program, ${ }^{2} \mathrm{Chengdu} \mathrm{has}$ provided free folic acid ( $400 \mu \mathrm{g} /$ day) for women of childbearing age who reside in the area with a plan to be pregnancy since 2010. The information regarding maternal folic use, prenatal exposures, perinatal health care and birth outcomes are prospectively collected by the Chengdu Maternal and Infant Health Surveillance system (CMIHS). These data allowed us to examine additional effect of maternal FAS on NsOC subtypes by performing a population-based case-control study.

\section{Results}

\section{General characteristics of the study subjects}


During October 2010 to September 2015, a total of 807 singletons with nonsyndromic clefts from CMIHS were available for the current analysis, including 247 NsCLs, 369 NsCLPs, and 191 NsCPs. Table 1 shows the maternal and infantile characteristics of the cases and 8070 controls. Compared with the control mothers, more NsCL/P mothers were under the age of 25 years or older than 35 years of age at the time of delivery. NsCL/P mothers were less educated ( $\leq 9$ years), or more overweighted $(\mathrm{BMI} \geq 24.0)$ than control mothers. Much more NsCL/P mothers exposed to environmental risks, and had a family history than control mothers. Male predominance in NsCL/P and female excess in NsCP were identified. No significant difference was found between cases and controls regarding the distribution of parity, and maternal medical conditions in the first trimester. 
Table 1

General characteristics of the study subjects

\begin{tabular}{|c|c|c|c|c|c|}
\hline \multirow[t]{2}{*}{ Variables } & Control & $\mathrm{NsCL}$ & NsCLP & NsCL/P & NsCP \\
\hline & $(n=8070)$ & $(n=247)$ & $(n=369)$ & $(n=616)$ & $(n=191)$ \\
\hline \multicolumn{6}{|c|}{ Maternal Age (years) ${ }^{a}$} \\
\hline$<25$ & $3058(37.9 \%)$ & 95 (38.5\%) & $156(42.3 \%)$ & $251(40.8 \%)$ & $65(34.0 \%)$ \\
\hline $25-34$ & 4495 (55.7\%) & $130(52.6 \%)$ & $175(47.4 \%)$ & 305 (49.5\%) & $111(58.1 \%)$ \\
\hline$>=35$ & $517(6.4 \%)$ & $22(8.9 \%)$ & 38 (10.3\%) & $60(9.7 \%)$ & $15(7.9 \%)$ \\
\hline \multicolumn{6}{|c|}{ Maternal Education (years) ${ }^{a}$} \\
\hline$<=9$ & $2004(24.9 \%)$ & $71(28.7 \%)$ & $101(27.4 \%)$ & 172 (27.9\%) & $44(23.0 \%)$ \\
\hline $10-13$ & $2689(33.3 \%)$ & $93(37.7 \%)$ & $133(36.0 \%)$ & $226(36.7 \%)$ & 68 (35.6\%) \\
\hline$>=13$ & 3377 (41.8\%) & $83(33.6 \%)$ & $135(36.6 \%)$ & 218 (35.4\%) & $79(41.4 \%)$ \\
\hline \multicolumn{6}{|c|}{ Urban-rural classification } \\
\hline Urban & $2972(36.8 \%)$ & $74(30.0 \%)$ & $106(28.7 \%)$ & $180(29.2 \%)$ & 73 (38.2\%) \\
\hline Rural & $5098(63.2 \%)$ & 173 (70.0\%) & $263(71.3 \%)$ & $436(70.8 \%)$ & $118(61.8 \%)$ \\
\hline \multicolumn{6}{|c|}{ Residence registration } \\
\hline Local & $7574(93.9 \%)$ & $233(94.3 \%)$ & $344(93.2 \%)$ & 577 (93.7\%) & $175(91.6 \%)$ \\
\hline Temporary & $496(6.1 \%)$ & $14(5.7 \%)$ & $25(6.8 \%)$ & 39 (6.3\%) & $16(8.4 \%)$ \\
\hline \multicolumn{6}{|c|}{ Maternal BMI $\left(\mathrm{kg} / \mathrm{m}^{2}\right)^{a}$} \\
\hline$<18.5$ & $1216(15.1 \%)$ & $36(14.6 \%)$ & 45 (12.2\%) & $81(13.2 \%)$ & $22(11.5 \%)$ \\
\hline $18.5-23.9$ & $5417(67.1 \%)$ & 145 (58.7\%) & 247 (66.9\%) & 392 (63.6\%) & $130(68.1 \%)$ \\
\hline$>=24$ & $1437(17.8 \%)$ & $66(26.7 \%)$ & $77(20.9 \%)$ & $143(23.2 \%)$ & $39(20.4 \%)$ \\
\hline \multicolumn{6}{|l|}{ Parity } \\
\hline Primiparous & $5342(66.2 \%)$ & 155 (62.8\%) & $260(70.5 \%)$ & 415 (67.4\%) & $124(64.9 \%)$ \\
\hline Multiparous & $2728(33.8 \%)$ & $92(37.2 \%)$ & 109 (29.5\%) & $201(32.6 \%)$ & $67(35.1 \%)$ \\
\hline \multicolumn{6}{|c|}{ Medical condition in the first trimester } \\
\hline No & $7406(91.8 \%)$ & $227(91.9 \%)$ & $335(90.8 \%)$ & $562(91.2 \%)$ & $183(95.8 \%)$ \\
\hline Yes & $664(8.2 \%)$ & $20(8.1 \%)$ & $34(9.2 \%)$ & $54(8.8 \%)$ & $8(4.2 \%)$ \\
\hline \multicolumn{6}{|c|}{ Environmental exposure in the first trimester } \\
\hline No & $7901(97.9 \%)$ & $243(98.4 \%)$ & $353(95.7 \%)$ & $596(96.8 \%)$ & $186(97.4 \%)$ \\
\hline Yes & $169(2.1 \%)$ & $4(1.6 \%)$ & $16(4.3 \%)$ & $20(3.2 \%)$ & $5(2.6 \%)$ \\
\hline
\end{tabular}

a Differed Significantly between controls and NsCL/Ps; ${ }^{\text {b }}$ Differed Significantly between controls and NsCPs; ${ }^{*}$ One NsCLP record with missing information on infant sex. 


\begin{tabular}{|llllll|}
\hline Variables & Control & NsCL & NsCLP & NsCL/P & NsCP \\
\cline { 2 - 6 } & $(\mathbf{n = 8 0 7 0 )}$ & $(\mathbf{n = 2 4 7 )}$ & $(\mathbf{n = 3 6 9 )}$ & $(\mathbf{n = 6 1 6 )}$ & $(\mathbf{n = 1 9 1 )}$ \\
\hline No & $8068(100.0 \%)$ & $242(98.0 \%)$ & $355(96.2 \%)$ & $597(96.9 \%)$ & $188(98.4 \%)$ \\
\hline Yes & $2(0.0 \%)$ & $5(2.0 \%)$ & $14(3.8 \%)$ & $19(3.1 \%)$ & $3(1.6 \%)$ \\
\hline Infant sex a,b,\# & & & & \\
\hline Female & $3919(48.6 \%)$ & $96(38.9 \%)$ & $145(39.3 \%)$ & $241(39.1 \%)$ & $109(57.1 \%)$ \\
\hline Male & $4151(51.4 \%)$ & $151(61.1 \%)$ & $223(60.4 \%)$ & $374(60.7 \%)$ & $82(42.9 \%)$ \\
\hline Gestational age (weeks) a,b & & & & & \\
\hline$<37$ & $406(5.0 \%)$ & $132(53.4 \%)$ & $269(72.9 \%)$ & $401(65.1 \%)$ & $17(8.9 \%)$ \\
\hline$>=37$ & $7664(95.0 \%)$ & $115(46.6 \%)$ & $100(27.1 \%)$ & $215(34.9 \%)$ & $174(91.1 \%)$ \\
\hline $\begin{array}{l}\text { a Differed Significantly between controls and NsCL/Ps; }{ }^{b} \text { Differed Significantly between controls and NsCPs; }{ }^{\#} \text { One } \\
\text { NsCLP record with missing information on infant sex. }\end{array}$ & & & \\
\hline
\end{tabular}

\section{Maternal periconceptional FAS}

As shown in Table 2, about $91 \%$ of control mothers and $81 \%$ case mothers had FAS during periconceptional period. More NsCL/Ps and NsCPs mothers did not take FA than control mothers (20.6\%, $12.5 \%$ vs $9.2 \%)$. Among mothers who had FAS, $23.7 \%$ of control mothers, $21.3 \%$ of NsCL/Ps mothers, and $17.3 \%$ of NsCPs mothers initiated FAS before LMP. In addition, pregnant women greater than 35 years of age, with lower education level, or with $\mathrm{BMI} \geq 24.0$, were more likely not to have FAS.

Table 2

Maternal periconceptional folic acid supplementation in cases and controls

\begin{tabular}{|llllll|}
\hline Group & Control & NsCL & NsCLP & NsCL/P & NsCP \\
\cline { 2 - 6 } & $(\mathbf{n = 8 0 7 0 )}$ & $(\mathbf{n = 2 4 7 )}$ & $(\mathbf{n = 3 6 9 )}$ & $\mathbf{( n = 6 1 6 )}$ & $(\mathbf{n = 1 9 1 )}$ \\
\hline Non-user & $743(9.2 \%)$ & $52(21.0 \%)$ & $75(20.3 \%)$ & $127(20.6 \%)$ & $24(12.5 \%)$ \\
\hline Periconception user & $7327(90.8 \%)$ & $195(79.0 \%)$ & $294(79.7 \%)$ & $489(79.4 \%)$ & $167(87.5 \%)$ \\
\hline Preconception user & $1909(23.7 \%)$ & $61(24.7 \%)$ & $70(19.0 \%)$ & $131(21.3 \%)$ & $33(17.3 \%)$ \\
\hline Post-Conception user & $5418(67.1 \%)$ & $134(54.3 \%)$ & $224(60.7 \%)$ & $358(58.1 \%)$ & $134(70.2 \%)$ \\
\hline Significant differences were found between controls, the overall and each subtype of nonsyndromic oral clefts. \\
\hline
\end{tabular}

\section{Association of maternal FAS and NsOC}

As shown in Table 3, the crude odds ratio of maternal periconceptional FAS for NsCL/P was 0.39 (95\% Cl: 0.32-0.48), and the adjusted OR was $0.41(95 \% \mathrm{Cl}: 0.33-0.51)$. Further stratification analysis showed that the associations remained significant for NsCL (aOR $=0.42,95 \%$ Cl: $0.30-0.58)$ and NsCLP (aOR = 0.41, 95\% Cl: 0.31-0.54). When analyzing according to the initiating time of maternal FAS, both maternal preconception and post-conception use could reduce the risks of NsCL/P and the subtypes (Table 3). Notably, maternal preconception FAS appeared to lower the risk of NsCP (aOR $=0.52,95 \% \mathrm{Cl}: 0.30-0.90)$. But the association between maternal periconceptional FAS and NsCP was nonsignificant. Several known risk factors such as advanced maternal age, higher maternal BMI, living in rural areas, exposure to environmental risks, nulliparity and male infants were positively associated with NsCL/P. Maternal illness in the first trimester and females were identified as risk factors of NsCP (Supplementary Table 1). 
Table 3

The associations between maternal peri-conceptional folic acid supplementation and nonsyndromic orofacial clefts

\begin{tabular}{|c|c|c|c|c|c|c|}
\hline \multirow{2}{*}{$\begin{array}{l}\text { Cleft } \\
\text { group }\end{array}$} & \multicolumn{2}{|c|}{ Periconception user } & \multicolumn{2}{|c|}{ Preconception user } & \multicolumn{2}{|c|}{ Post-conception user } \\
\hline & cOR $(95 \% \mathrm{Cl})$ & aOR $(95 \% \mathrm{Cl})$ & cOR $(95 \% \mathrm{Cl})$ & aOR $(95 \% \mathrm{Cl})$ & cOR $(95 \% \mathrm{Cl})$ & aOR $(95 \% \mathrm{Cl})$ \\
\hline $\mathrm{NsCL} / \mathrm{P}$ & $0.39(0.32,0.48)$ & $0.41(0.33,0.51)$ & $0.40(0.31,0.52)$ & $0.43(0.33,0.56)$ & $0.39(0.31,0.48)$ & $0.41(0.33,0.51)$ \\
\hline $\mathrm{NsCL}$ & $0.38(0.28,0.53)$ & $0.42(0.30,0.58)$ & $0.46(0.31,0.67)$ & $0.51(0.34,0.75)$ & $0.35(0.26,0.50)$ & $0.39(0.28,0.55)$ \\
\hline NsCLP & $0.40(0.31,0.52)$ & $0.41(0.31,0.54)$ & $0.36(0.26,0.51)$ & $0.38(0.27,0.54)$ & $0.41(0.31,0.54)$ & $0.42(0.32,0.56)$ \\
\hline $\mathrm{NsCP}$ & $0.71(0.47,1.12)$ & $0.69(0.45,1.10)$ & $0.54(0.32,0.92)$ & $0.52(0.30,0.90)$ & $0.77(0.50,1.22)$ & $0.75(0.48,1.20)$ \\
\hline
\end{tabular}

\section{Discussion}

In this population-based case-control study, we demonstrated that maternal periconceptional FAS was associated with a reduced risk of overall $\mathrm{NsCL} / \mathrm{P}$, and the reduced risk varied by cleft subtype and supplementation initiation timing. We did not identify the preventive effect of periconceptional FAS for NsCP, whereas we observed a significant association between maternal FAS started before LMP and NsCP, suggesting that earlier or longer supplementation may be protective.

Consistent with previous studies in some western ${ }^{9-17}$ and Asian non-Chinese populations, ${ }^{19,20}$ our results showed that maternal periconceptional FAS could reduce the risk of overall NsCL/P by approximately $60 \%$ regardless of the initiation timing. This preventive effect was observed for both NsCL and NsCLP. Similar associations have been noted in several epidemiological studies in China. ${ }^{18,31-35}$ These investigations were hospital-based and mainly focused on the environmental risk factors rather than the effect of supplementation. So far, only one cohort study specifically evaluated the preventive effect of maternal FAS on oral clefts, and found that in the north of China maternal supplementation started before the LMP reduced the risk of $\mathrm{NsCL} / \mathrm{P}(\mathrm{NsCL} / \mathrm{P}, \mathrm{aRR}=0.69,95 \% \mathrm{Cl} 0.55-0.87$; NsCL, $\mathrm{aRR}=0.26,95 \% \mathrm{Cl}$ : 0.07-0.98; NsCLP, aRR $=0.19,95 \% \mathrm{Cl}: 0.07-0.50$ ), while such effect was neither observed in the south of China nor for supplementation started on or after the LMP. ${ }^{18,32-36}$ Another population-based case-control study in Shaanxi province reported a preventive effect of optimal FAS for overall birth defects $(\mathrm{OR}=0.71,95 \% \mathrm{Cl}: 0.57-0.89)$, but not for oral clefts. ${ }^{26}$ The authors thought that non-randomized maternal FA use, small sample size and supplementation initiation timing could explain differences studies. ${ }^{18,26}$ A recent meta-analysis revealed the preventive effect of maternal FAS in early pregnancy against NsCL/P and NsCP, and identified publication bias in previously published researches. ${ }^{7}$ However, findings from randomised or quasi-randomised trials seemed not to support the preventive effect of daily FAS on oral clefts. ${ }^{31}$ It can be seen that inconsistences among various studies are obvious. In fact, there is still a lack of reliable evidence on the preventive effect of folic acid on oral clefts, especially on the dose and initiation timing of supplementation.

Nonsyndromic cleft palate has been regarded as a distinct condition from NsCL/P on embryologic origin and etiology. As noted in previous investigation, $6,11,18,21,32$ this study found a negative but nonsignificant association of maternal periconceptional FAS with NsCP. Interestingly, maternal FAS starting before LMP was significantly associated with decreased risk of NsCP, suggesting that earlier use may work or supplementation initiation timing may play a part. A few studies reported the preventive effect of FAS on NsCP. ${ }^{7,33}$ Compared with NsCL/P, researches on FAS and NsCP produced more conflicting results. A cohort study in Norway reported an adjusted RR of 0.84 (95\% Cl: $0.66-1.06)$ for maternal use of Vitamins/folic acid and NsCP, and a stronger association ( $\mathrm{RR}=0.63,95 \% \mathrm{Cl}: 0.45-0.88)$ for cleft palate in combination with other malformations. ${ }^{24}$ On the contrary, a case-control study conducted in Northern Netherlands ${ }^{27}$ and another cohort study ${ }^{28}$ in Japan identified maternal FAS/multivitamin supplement use during the first trimester as a risk factor of NsCP. 
Whether the preventive effect of maternal FAS against NsCP can be observed in a certain population depends on various factors, including genetic background, maternal dietary folic acid intake, serum folate level and the compliance with FAS, etc. Considering the small number of NsCP cases in our analysis, the findings about maternal FAS and NsCP need to be further studied. These controversial results may be due to the heterogeneities in research design, population, sample size, exposure assessment, and other potential confounders., 18,21,32-36 Overall, more prospective studies are needed to elucidate the relationship between maternal FAS and NsCP.

Several strengths and limitations should be considered when interpreting our study results. The population-based nature and large sample size of Chinese cleft cases of the study ensure robust OR estimates for NsCL/P subtypes. We got the statistical power of 0.82 for NsCL and 0.98 for NsCLP. In addition, the prospectively collected exposure data could minimize recall bias. Though information of maternal dietary and multivitamin intake was not available for analysis, the associations are less likely to be distorted because the OR estimates were based on randomly selected control data with adjustment for known risk factors such as maternal illness, social factors, environmental exposures, and maternal illness.

In conclusion, our study provides additional evidence that maternal FAS during periconceptional period can reduce the risk of NsCL/P. Larger-sample-size studies are warranted to elucidate the association with NsCP and to determine whether women can benefit more from supplementation starting before LMP. It is of paramount importance for women of childbearing age to become aware of that maternal FAS can not only reduce the risk of NTD, but also reduce the risk of $\mathrm{NsCL} / \mathrm{P}$.

\section{Methods}

\section{Data source and study subjects}

Data for this study were abstracted from the CMIHS. According to the "Maternal and Infant Health Care Protocol of Sichuan Province", 37 every pregnant woman was required to have at least five prenatal medical examinations during her pregnancy and three postnatal clinical visits (on the 7th, 28th and 42nd day after delivery). The results of examinations or visits, risk factors (maternal diseases, family history, other medical conditions), and pregnancy outcomes (spontaneous abortion, stillbirth, live birth, birth defects, etc.), were recorded in the CMIHS system. The medical examination, data collecting, checking and auditing were performed by well-trained obstetricians and nurses. CMIHS adopted the diagnosis criterions, case ascertainment, quality assurance, data collection and encoding of birth defects proposed by Chinese Birth Defects Monitoring Network. ${ }^{38}$ Between October 2010 and September 2015, 807 singletons with NsOCs were included in as the cases, for each case ten controls were randomly selected from the healthy singletons born in the same period.

\section{Folic Acid Intake And Covariates}

Briefly, women who joined in the prevention program were followed once a month by local community healthcare workers, and their registration dates, last menstrual period (LMP), dates of starting and ending use, and the information of folic acid supplementation were recorded. In the CMIHS system, a woman's FAS information was linked to her medical records once she had her first prenatal exam in any of the local hospitals.

In this study, pregnant women who regularly took folic acid during the periconceptional period for at least one month were defined as "periconception users". Of them, those who started intake before their LMP were termed as "preconception users", while those who started on or after their LMP were named as "post-conception users". On the contrary, pregnant women who did not have folic acid intake, or took it continuously less than one month were considered as "non-users". Other variables included maternal age, nationality, education, residence, parity, medical condition and environmental exposures in the first trimester. Specifically, the maternal medical condition referred to any of such conditions as positive result of syphilis, human immunodeficiency virus and hepatitis B virus testing, anemia, chronic kidney, liver and heart 


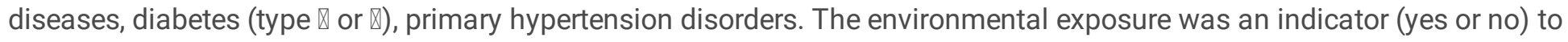
show whether the mothers had smoking, alcohol drinking, drug abuse, or exposure to radiation and hazardous substances. The variables of infants included gestational age, date of birth, birth weight, sex, birth defects and infant outcomes. Data used in this study were anonymous and included no individually identifiable information. This research was approved by the Medical Ethics Committee of the West China Second University Hospital, Sichuan University. All methods were performed in accordance with relevant regulations and the individual informed consent was waived because the study used non-identified data.

\section{Statistical Analyses}

Differences in maternal and infant characteristics between cases and controls were examined with independent sample ttests for continuous variables and with Pearson chi-square tests for categorical variables. Non-conditional logistic regression analysis was used to calculate the crude and adjusted odds ratios (ORs) and 95\% confidence intervals (Cl). Statistical analyses were performed with R 3.5.3 (R Development Core Team 2019) and packages "rms".

\section{Declarations}

\section{Acknowledgements}

This work was supported by the Grants from National Key R\&D Program of China (2017YFC0907304) and National Natural Foundation of China (81072375).

\section{Author contributions}

WX performed statistical work and drafted the manuscript. LY, CD, ZZ, LR, ZR, SZ, TZ and GZ participated in the data collection and preparation. LR and GZ provided data access. HL and LD designed research plan and revised the manuscript. All authors had read and approved the final manuscript.

\section{Conflicts of interest}

The authors report no conflicts of interest. The authors alone are responsible for the content and writing of this article.

Funding: This work was supported by the Grants from National Key R\&D Program of China (2017YFC0907304), National Natural Foundation of China (81072375), and Ministry of Health.

Disclosure statement: The authors report no conflicts of interest. The authors alone are responsible for the content and writing of this article.

\section{References}

1. Mossey, P. A. \& Modell, B. Epidemiology of oral clefts 2012: an international perspective. Front Oral Bio/ 16, 1-18, doi:10.1159/000337464 (2012).

2. $\mathrm{MOH}$. National stocktaking report on birth defect prevention (2012). (Ministry of Health, 2012).

3. Wang, M. et al. Prevalence of Orofacial Clefts among Live Births in China: A Systematic Review and Meta-Analysis. Birth Defects Res 109, 1011-1019, doi:10.1002/bdr2.1043 (2017).

4. Dai, L. et al. Time trends in oral clefts in Chinese newborns: data from the Chinese National Birth Defects Monitoring Network. Birth Defects Res A Clin Mol Terato/ 88, 41-47, doi:10.1002/bdra.20607 (2010).

5. Tolarova, M. Periconceptional supplementation with vitamins and folic acid to prevent recurrence of cleft lip. Lancet $\mathbf{2}$, 217, doi:10.1016/s0140-6736(82)91063-7 (1982). 
6. Millacura, N., Pardo, R., Cifuentes, L. \& Suazo, J. Effects of folic acid fortification on orofacial clefts prevalence: a meta-analysis. Public health nutrition 20, 2260-2268, doi:10.1017/S1368980017000878 (2017).

7. Jahanbin, A., Shadkam, E., Miri, H. H., Shirazi, A. S. \& Abtahi, M. Maternal Folic Acid Supplementation and the Risk of Oral Clefts in Offspring. J Craniofac Surg 29, e534-e541, doi:10.1097/SCS.0000000000004488 (2018).

8. Zhou, Y. et al. Folate intake, markers of folate status and oral clefts: An updated set of systematic reviews and metaanalyses. Birth Defects Res 112, 1699-1719, doi:10.1002/bdr2.1827 (2020).

9. Czeizel, A. E. The primary prevention of birth defects: Multivitamins or folic acid? International journal of medical sciences 1, 50-61, doi:10.7150/ijms.1.50 (2004).

10. van Rooij, I. A. et al. Periconceptional folate intake by supplement and food reduces the risk of nonsyndromic cleft lip with or without cleft palate. Prev Med 39, 689-694, doi:10.1016/j.ypmed.2004.02.036 (2004).

11. Wilcox, A. J. et al. Folic acid supplements and risk of facial clefts: national population based case-control study. BMJ 334, 464, doi:10.1136/bmj.39079.618287.0B (2007).

12. Kelly, D., O'Dowd, T. \& Reulbach, U. Use of folic acid supplements and risk of cleft lip and palate in infants: a population-based cohort study. Br J Gen Pract 62, e466-472, doi:10.3399/bjgp12X652328 (2012).

13. Czeizel, A. E., Toth, M. \& Rockenbauer, M. Population-based case control study of folic acid supplementation during pregnancy. Teratology53, 345-351, doi:10.1002/(SICI)1096-9926(199606)53:6<345::AID-TERA5>3.0.C0;2-Z (1996).

14. Chevrier, C. et al. Fetal and maternal MTHFR C677T genotype, maternal folate intake and the risk of nonsyndromic oral clefts. Am J Med Genet A 143A, 248-257, doi:10.1002/ajmg.a.31462 (2007).

15. Carmichael, S. L. et al. Reduced risks of neural tube defects and orofacial clefts with higher diet quality. Archives of pediatrics \& adolescent medicine 166, 121-126, doi:10.1001/archpediatrics.2011.185 (2012).

16. Figueiredo, R. et al. The role of the folic acid to the prevention of orofacial cleft: an epidemiological study. Oral Diseases 21, 240-247 (2015).

17. Itikala, P. R., Watkins, M. L., Mulinare, J., Moore, C. A. \& Liu, Y. Maternal multivitamin use and orofacial clefts in offspring. Teratology 63, 79-86, doi:10.1002/1096-9926(200102)63:2<79::AID-TERA1013>3.0.C0;2-3 (2001).

18. Li, S. et al. Folic acid use and nonsyndromic orofacial clefts in China: a prospective cohort study. Epidemiology 23, 423-432, doi:10.1097/EDE.0b013e31824d0349 (2012).

19. Golalipour, M. J., Vakili, M. A. \& Kaviani, N. Reduction in non syndromic oral clefts following mandatory flour fortification with folic acid in Northern Iran. Medical journal of the Islamic Republic of Iran 28, 29 (2014).

20. Dien, V. H., McKinney, C. M., Pisek, A. \& Pitiphat, W. Maternal exposures and risk of oral clefts in South Vietnam. Birth defects research $110,527-537$ (2018).

21. Wang, W., Guan, P., Xu, W. \& Zhou, B. Risk factors for oral clefts: a population-based case-control study in Shenyang, China. Paediatr Perinat Epidemio/ 23, 310-320, doi:10.1111/j.1365-3016.2009.01025.x (2009).

22. Hashmi, S. S., Waller, D. K., Langlois, P., Canfield, M. \& Hecht, J. T. Prevalence of nonsyndromic oral clefts in Texas: 1995-1999. Am J Med Genet A 134, 368-372, doi:10.1002/ajmg.a.30618 (2005).

23. Gildestad, T. et al. Maternal use of folic acid and multivitamin supplements and infant risk of birth defects in Norway, 1999-2013. British Journal of Nutrition 124, 316-329 (2020).

24. Gildestad, T. et al. Folic acid supplements and risk for oral clefts in the newborn: a population-based study. British Journal of Nutrition 114, 1456-1463 (2015).

25. Godwin, K. A. et al. Changes in frequencies of select congenital anomalies since the onset of folic acid fortification in a Canadian birth defect registry. Canadian journal of public health = Revue canadienne de sante publique 99, 271-275 (2008).

26. Qu, P. et al. A propensity-matched study of the association between optimal folic acid supplementation and birth defects in Shaanxi province, Northwestern China. Scientific reports9, 5271, doi:10.1038/s41598-019-41584-5 (2019). 
27. Rozendaal, A. M. et al. Periconceptional folic acid associated with an increased risk of oral clefts relative to non-folate related malformations in the Northern Netherlands: a population based case-control study. Eur J Epidemio/ 28, 875887, doi:10.1007/s10654-013-9849-0 (2013).

28. Yoshida, S., Takeuchi, M., Kawakami, C., Kawakami, K. \& Ito, S. Maternal multivitamin intake and orofacial clefts in offspring: Japan Environment and Children's Study (JECS) cohort study. BMJ open 10, e035817 (2020).

29. Johnson, C. Y. \& Little, J. Folate intake, markers of folate status and oral clefts: is the evidence converging? International journal of epidemiology 37, 1041-1058, doi:10.1093/ije/dyn098 (2008).

30. De-Regil, L. M., Fernandez-Gaxiola, A. C., Dowswell, T. \& Pena-Rosas, J. P. Effects and safety of periconceptional folate supplementation for preventing birth defects. The Cochrane database of systematic reviews, CD007950, doi:10.1002/14651858.CD007950.pub2 (2010).

31. De-Regil, L. M., Pena-Rosas, J. P., Fernandez-Gaxiola, A. C. \& Rayco-Solon, P. Effects and safety of periconceptional oral folate supplementation for preventing birth defects. The Cochrane database of systematic reviews, CD007950, doi:10.1002/14651858.CD007950.pub3 (2015).

32. Hao, Y. et al. Association of Parental Environmental Exposures and Supplementation Intake with Risk of Nonsyndromic Orofacial Clefts: A Case-Control Study in Heilongjiang Province, China. Nutrients 7, 7172-7184, doi:10.3390/nu7095328 (2015).

33. Jia, Z. L. et al. Maternal malnutrition, environmental exposure during pregnancy and the risk of non-syndromic orofacial clefts. Oral Dis 17, 584-589, doi:10.1111/j.1601-0825.2011.01810.x (2011).

34. Xu, D. P. et al. A Study on Environmental Factors for Nonsyndromic Cleft Lip and/or Palate. J Craniofac Surg 29, 364367, doi:10.1097/SCS.0000000000004214 (2018).

35. Xu, L. F. et al. A Case-control Study of Environmental Risk Factors for Nonsyndromic Cleft of the Lip and/or Palate in Xuzhou, China. Biomed Environ Sci 28, 535-538, doi:10.3967/bes2015.076 (2015).

36. Lin, Y., Shu, S. \& Tang, S. A case-control study of environmental exposures for nonsyndromic cleft of the lip and/or palate in eastern Guangdong, China. Int J Pediatr Otorhinolaryngo/ 78, 544-550, doi:10.1016/j.ijporl.2014.01.002 (2014).

37. PU Jie, Z. S.-j., QIAN Ping. Survey on Awareness and Demand for Preconception Care of Womenin Chengdu. West China Medical Journal 28, 1055-1058 (2013).

38. Zhu, P., Du, X. \& Li, M. Prevalence of low birthweight and its influencing factors from 2008 to 2014 in Chengdu. Modern Preventive Medicine 44, 1428-1432 (2017).

\section{Supplementary Files}

This is a list of supplementary files associated with this preprint. Click to download.

- SupplementaryTable1MaternalpericonceptionalfolicacidsupplementationandtheriskofNSOFC.docx 